\title{
Inhaled Nitric Oxide Decreases Leukocyte Trafficking in the Neonatal Mouse Lung During Exposure to $>95 \%$ Oxygen
}

\author{
MELISSA J. ROSE, MICHAEL R. STENGER, MANDAR S. JOSHI, STEPHEN E. WELTY, JOHN ANTHONY BAUER, \\ AND LEIF D. NELIN \\ Center for Perinatal Research [M.R.S., S.E.W., L.D.N.] and Center for Cardiovascular Medicine [M.J.R., M.S.J., J.A.B.], The Research \\ Institute at Nationwide Children's Hospital, Columbus, Ohio 43205
}

\begin{abstract}
Chronic lung injury in the neonate is termed bronchopulmonary dysplasia (BPD). These patients generally require supplemental oxygen therapy, and hyperoxia has been implicated in the pathogenesis of BPD. The concomitant use of oxygen and inhaled NO (iNO) may result in the generation of reactive nitrogen species or may have an anti-inflammatory effect in the neonatal lung. We tested the hypothesis that exposure to $>95 \% \mathrm{O}_{2}$ in neonatal mice would increase trafficking of leukocytes into the lung and that the addition of iNO to $>95 \% \mathrm{O}_{2}$ would decrease this leukocyte trafficking. Hyperoxia resulted in fewer alveoli, increased presence of neutrophils and macrophages, and decreased number of mast cells within the lung parenchyma. Adding iNO to hyperoxia prevented the hyperoxia-induced changes and resulted in the numbers of alveoli, neutrophils, macrophages, and mast cells approximating those found in controls (room air exposure). Intercellular adhesion molecule (ICAM) and monocyte chemotactic protein-1 (MCP-1), two factors responsible for leukocyte recruitment, were up-regulated by hyperoxic exposure, but the addition of iNO to the hyperoxic exposure prevented the hyperoxia-induced up-regulation of ICAM and MCP-1. These data demonstrate that iNO alters the hyperoxia-induced recruitment of leukocytes into the lung. (Pediatr Res 67: 244-249, 2010)
\end{abstract}

$\mathrm{E}^{\mathrm{x}}$ xposure to supplemental oxygen commonly results in the local production of proinflammatory cytokines, recruitment of leukocytes, and oxidative tissue injury (1-4). However, supplemental oxygen can be life saving in the intensive care unit setting. Hyperoxic exposure in the neonatal period is associated with arrested or delayed development of postnatal lung maturation (5). The specific mechanisms underlying hyperoxia-induced arrested lung development are not completely defined, although increased oxidative stress and activation of the inflammatory cascade, as well as infiltration of leukocytes into the lungs are recognized as important contributors. These factors are also thought to contribute to the pathogenesis of chronic neonatal lung diseases, such as bronchopulmonary dysplasia (BPD).

Inhaled NO (iNO), a selective pulmonary vasodilator, has been used in term and near-term infants with pulmonary

Received September 14, 2009; accepted October 24, 2009

Correspondence: Leif D. Nelin, M.D., The Research Institute at Nationwide Children's Hospital, 700 Children's Drive-W203, Columbus, OH 43205; e-mail: Leif.Nelin@ nationwidechildrens.org

Supported in part by a National Heart Lung and Blood Institute Grant HL-075261. The NO gas and the aeromax delivery system were provided by a grant to L.D.N. from Ikaria (Clinton, NJ). hypertension (6-8). Recently, the clinical use of iNO for the prevention and/or treatment of BPD have been suggested (9). Supplemental $\mathrm{O}_{2}$ therapy is commonly used in combination with iNO in the intensive care unit; however, their mechanistic and physiologic interactions in vivo are not completely understood. For example, in biologic settings NO is known to avidly interact with reactive oxygen species to generate reactive nitrogen species such as peroxynitrite, a potent oxidizing agent that can cause cellular injury and death. In contrast, iNO has also been shown to prevent tissue injury in the lung (9). Thus, the interplay between supplemental $\mathrm{O}_{2}$ therapy and iNO may result in a wide range of physiologic effects, and furthermore, the net effect may be either beneficial or deleterious in any given physiologic setting. A better understanding of these interactions is necessary to improve patient outcomes and to tailor iNO therapy for specific clinical settings. The goals of this study, therefore, were to investigate the interactions of hyperoxia and iNO with regards to leukocyte trafficking in vivo. Using a neonatal mouse model, we tested the hypothesis that recruitment of leukocytes into the lungs is increased in hyperoxia and that adding a therapeutically relevant dose of iNO to the hyperoxic exposure would prevent the recruitment of leukocytes into the lungs.

\section{METHODS}

Animal model. Time-dated pregnant FVB mice $(n=36)$ were purchased from Charles River (Wilmington, MA) and were maintained in the animal care facility of the Research Institute at Nationwide Children's Hospital. All animals were kept in polycarbonate cages with wire lids and $100 \%$ aspen wood chip bedding. The animals were allowed free access to food and water, and a 12:12-h day:night cycle was maintained throughout the study. The Institutional Animal Care and Use Committee of the Research Institute at Nationwide Children's Hospital approved all experimental protocols.

Oxygen/NO exposure. The exposures were done as previously described (5). Dams delivered naturally at term. Within $24 \mathrm{~h}$ of birth, the cage containing the mother and her pups was placed in a Plexiglas chamber. The $>95 \% \mathrm{O}_{2}$ atmosphere was maintained with an $\mathrm{O}_{2}$ flow of $\sim 10 \mathrm{~L} / \mathrm{min}$ through the chamber. For the iNO exposure groups, 800 parts per million (ppm) iNO (Ikaria, Port Allen, LA) was delivered at $\sim 125 \mathrm{~mL} / \mathrm{min}$. Soda lime (Fisher Scientific, Fair Lawn, NJ) was present in the chamber during all exposures to absorb $\mathrm{CO}_{2}$ and $\mathrm{NO}_{2}$. Each litter was exposed for $72 \mathrm{~h}$ to: 1) $21 \% \mathrm{O}_{2}$ [room air (RA); $n=9$ ], 2) $21 \% \mathrm{O}_{2}+10 \mathrm{ppm}$ iNO (RA + iNO; $n=9$ ), 3) $>95 \%$ $\mathrm{O}_{2}(n=7)$, or 4$)>95 \% \mathrm{O}_{2}+10 \mathrm{ppm}$ iNO $(n=11)$. The $\mathrm{O}_{2}$ and $\mathrm{CO}_{2}$ levels

Abbreviations: BPD, bronchopulmonary dysplasia; H\&E, hematoxylin and eosin; ICAM, intercellular adhesion molecule; iNO, inhaled NO; MCP-1, monocyte chemotactic protein-1; MPO, myeloperoxidase; ppm, parts per million; RA, room air 
A

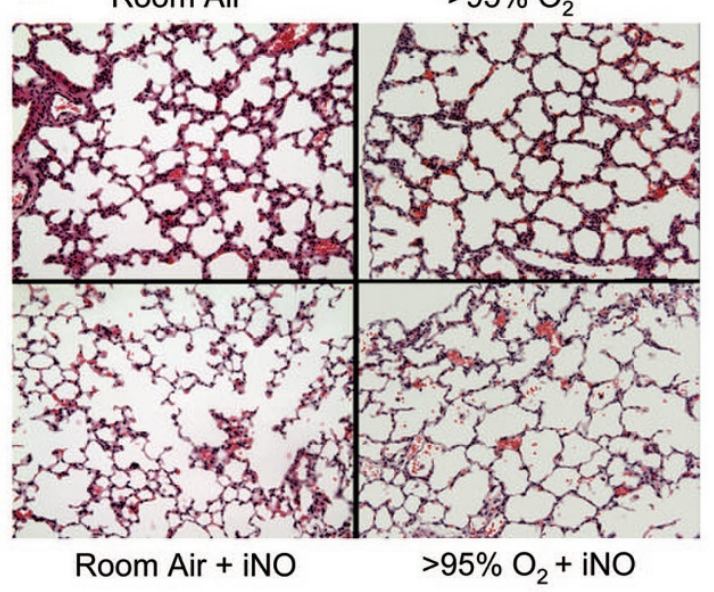

B

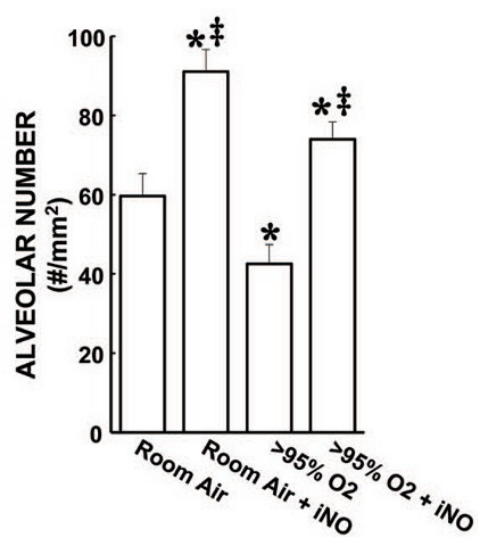

Figure 1. Hyperoxia-induced reduction in alveolar numbers in neonatal mouse lungs is prevented by the addition of iNO. A, Representative H\&E stained images (100× magnification) of alveolar regions from the lungs of mice exposed for $72 \mathrm{~h}$ to each of the four treatment groups. $B$, The mean \pm SEM of the alveolar numbers from all of the lung sections examined. $*$ Different from RA, $p<0.05$. $\ddagger$ Different from $>95 \% \mathrm{O}_{2}, p<0.05$. in the exposure chamber were measured at least twice daily (AEI Technologies, Pittsburgh, $\mathrm{PA}$ ). The $\mathrm{NO}$ and $\mathrm{NO}_{2}$ levels in the exposure chamber were measured at least four times a day (Aeronox, Ikaria, Clinton, NJ). For each exposure group, a RA control group was exposed concurrently, and the dams were switched between the exposure group and the RA controls every $24 \mathrm{~h}$. Animals were killed at $24 \mathrm{~h}$ for mRNA and at $72 \mathrm{~h}$ for immunohistochemistry and tissue morphometry. Mice were killed with an injection of pentobarbital $(100 \mathrm{mg} / \mathrm{kg})$ i.v. The small size of the lungs precluded doing multiple measures on any given lung tissue, therefore a different time point was chosen for each methodology. We chose to perform mRNA at $24 \mathrm{~h}$ because changes in mRNA would be expected to occur first.

Real time PCR. Total mRNA was isolated from the lungs of mice in the four exposure groups ( $n=3$ in each group) using Trizol reagent. The mRNA was treated with RQ1 RNase-free DNase (Promega) to eliminate genomic DNA contamination and reverse transcription done using avian myeloblastosis virus-reverse transcriptase. Real-time PCR reactions contained $12.5 \mu \mathrm{L}$ of JumpStart Taq ReadyMix (Sigma Chemical Co.-Aldrich Inc.), $0.4 \mu \mathrm{M}$ of forward and reverse primers, and $1 \mu \mathrm{L}$ of template cDNA. No template control and no reverse transcription control were used as negative controls. The $18 \mathrm{~S}$ was used as the reference gene. Intercellular adhesion molecule (ICAM)-1 was amplified using forward primer 5'-ACA CTA TGT GGA CTG GCA GTG GTT-3' and reverse primer 5'-TGA GGC TCG ATT GTT CAG CTG CTA-3'. Monocyte chemotactic protein-1 (MCP-1) was amplified using forward primer $5^{\prime}$-AGC AGG TGT CCC AAA GAA GCT GTA-3' and reverse primer 5'-AAA GGT GCT GAA GAC CTT AGG GCA-3'. The 18S was amplified using forward primer $5^{\prime}$-CCA GAG CGA AAG CAT TTG CCA AGA-3' and reverse primer $5^{\prime}$-TCG GCA TCG TTT ATG GTC GGA ACT- $3^{\prime}$. The Real-time PCR mixture was denatured at $94^{\circ} \mathrm{C}$ for 2 min, and then cycled at denaturing temperature of $94^{\circ} \mathrm{C}$ for $15 \mathrm{~s}$, annealing temperature of $60^{\circ} \mathrm{C}$ for $1 \mathrm{~min}$, extension temperature of $72^{\circ} \mathrm{C}$ for $1 \mathrm{~min}$ for a total of 40 cycles. A melting curve program was run immediately after the PCR program to make sure the desired amplicon was detected. The PCR reaction was performed using Chromo4 System for Real-time PCR detection (Bio-Rad). The $\Delta \Delta \mathrm{C}_{\mathrm{t}}$ method was used to calculate the fold change in mRNA expression: $\Delta \mathrm{C}_{\mathrm{t}}=\mathrm{C}_{\mathrm{t}}$ (target gene) $-\mathrm{C}_{\mathrm{t}}$ (housekeeping gene), $\Delta \Delta \mathrm{C}_{\mathrm{t}}=\Delta \mathrm{C}_{\mathrm{t}}$ (treatment) $-\Delta \mathrm{C}_{\mathrm{t}}$ (control), fold change $=2^{(-\Delta \Delta \mathrm{Ct})}$.

Tissue morphometry and immunohistochemistry. Animals were killed by an intraperitoneal injection of pentobarbital sodium $(100 \mathrm{mg} / \mathrm{kg})$. The tracheas were cannulated with a 25 -gauge Silastic catheter, and $10 \%$ neutral buffered formalin was instilled at $25 \mathrm{~cm} \mathrm{H}_{2} \mathrm{O}$ pressure. The trachea was then tied and lungs removed and fixed overnight in $10 \%$ neutral buffered formalin. The next day, lungs were washed in PBS five times, transferred to PBS, and stored at $4^{\circ} \mathrm{C}$. Lungs were serially dehydrated in increasing concentrations of ethanol and then embedded in paraffin. For preparation of paraffin blocks, left lungs were cut transversely (perpendicular to craniocaudal axis) at the level of the left main bronchus. The cut slices of lung tissue were identically oriented and made into paraffin blocks. Sections of lung tissues $(5 \mu \mathrm{m})$ were deparaffinized for immunostaining.

Using serial sections, tissues were stained with hematoxylin and eosin (H\&E) and immunostained with antibodies against, myeloperoxidase (MPO) (1:100 dilution; $200 \mu \mathrm{g} / \mathrm{mL}$ antibody protein concentration, Neomarkers), CD68 (1:800; $435 \mu \mathrm{g} / \mathrm{mL}$, Dako), ICAM (1:2000; $200 \mu \mathrm{g} / \mathrm{mL}$, Santa Cruz), and MCP-1 (1:100; $200 \mu \mathrm{g} / \mathrm{mL}$, Santa Cruz). Staining control tissues were exposed for the same duration to nonimmune goat $\mathrm{IgG}$ for MPO, nonimmune mouse $\mathrm{IgG}$ for $\mathrm{CD} 68$, and ICAM and nonimmune rabbit IgG for MCP-1, in place of primary antibody. Diaminobenzidine $(0.06 \% \mathrm{wt} / \mathrm{vol})$ was used to provide visualization of immunoreactivity followed by methyl green counterstaining to identify nuclei. Mast cell and eosinophil staining was achieved with Toluidine Blue $\mathrm{O}$ and Vital New Red solutions, respectively.

After staining, identically oriented tissue sections were visualized with an Olympus Optical (New York, NY) BX-40 microscope (800× magnification) and captured under identical lighting conditions and optical settings using an Insight digital camera. Images were analyzed using digital image analysis software (Image Pro Plus 4.0; Media Cybernetics, Silver Spring, MD) as previously described (5). Alveolar morphometry was performed on tissues stained with H\&E. Automated identification of alveoli was achieved by defining an alveolus as an enclosed region of tissue with a minimal area of 400 $\mu \mathrm{m}^{2}$. The number of alveoli was computed from 35 to 60 images from four to eight animals per treatment group. Images were segmented to eliminate background and nuclear counterstain while capturing tissue staining, allowing tissue area per image to be computed. Cells with positive staining for MPO, CD68, Toluidine Blue O solution, Vital New Red solution, and MCP-1 were manually noted on 60-170 images taken from four to eight animals per treatment group. The investigator evaluating positively stained cells was blinded to exposure conditions. Using this procedure, we have previously found that the intraobserver and interobserver variability to be $<5 \%$ and $<10 \%$, respectively (5). To analyze the ICAM-1 staining, the OD measurements were multiplied by the total positive stained tissue area to give an IOD, a measure of staining intensity (5).

Statistical Analysis. All results are shown as the mean \pm SEM. Comparisons between treatment groups were made using one-way ANOVA with differences identified using a Newman-Keuls posthoc test. Spearman's nonparametric correlation analyses were performed to investigate relationships between two measured variables across treatment groups. All analyses were performed using STATA statistical software and in all cases differences were considered significant when $p<0.05$.

\section{RESULTS}

Morphometry. Shown in Figure $1 A$ are representative H\&E stained images of alveolar regions from each of the four treatment groups. Alveolar numbers were determined using automated digital image analyses, using 146 individual images. The mean values for alveolar number in each treatment group are shown in Figure $1 B$. The lungs from animals exposed to RA for $72 \mathrm{~h}$ had an average of $\sim 60$ alveoli per $\mathrm{mm}^{2}$. The lungs from animals exposed to RA + iNO for $72 \mathrm{~h}$ had significantly more alveoli than did the lungs from animals exposed to RA alone (Fig. $1 B$ ). The lungs from animals exposed to $>95 \% \mathrm{O}_{2}$ for $72 \mathrm{~h}$ had significantly fewer alveoli than did the lungs from RA controls. The addition of iNO to the $>95 \% \mathrm{O}_{2}$ exposure resulted in alveolar numbers that were significantly greater after $72 \mathrm{~h}$ of exposure than those found in 
either the lungs from animals exposed to $>95 \% \mathrm{O}_{2}$ or the lungs from the RA controls (Fig. 1B).

Leukocyte prevalence. There was $\sim 2.5$-fold more neutrophils in the lungs from animals exposed to $>95 \% \mathrm{O}_{2}$ for $72 \mathrm{~h}$ than in the lungs from RA-exposed animals (Fig. 2). Interestingly, in the lungs from the RA + iNO group there were significantly fewer neutrophils than in the lungs from RA controls (Fig. 2). The addition of $10 \mathrm{ppm}$ iNO to the $>95 \% \mathrm{O}_{2}$ exposure prevented the hyperoxia-induced increase in neutrophil numbers, such that the iNO $+>95 \% \mathrm{O}_{2}$ group was not different from RA controls. There were macrophages identified in the lungs from RA-exposed animals, and addition of iNO to the RA exposure had no significant effect on macrophage numbers (Fig. $3 A$ ). In the lungs from animals exposed to $>95 \% \mathrm{O}_{2}$ for $72 \mathrm{~h}$ there was $\sim 3$ times as many macrophages as in the lungs from animals exposed to RA (Fig. 3A). The addition of iNO to the $>95 \% \mathrm{O}_{2}$ exposure prevented the hyperoxia-induced increase in macrophage numbers (Fig. $3 A$ ).
In contrast to the effects observed for MPO + and $\mathrm{CD} 68+$ cells, exposure to $>95 \% \mathrm{O}_{2}$ alone for $72 \mathrm{~h}$ resulted in significantly fewer alveolar mast cells in the lung (Fig. 3B). Furthermore, the addition of iNO to either RA or $>95 \% \mathrm{O}_{2}$ had no significant effect on mast cell prevalence (Fig. $3 B$ ). There was greater variation in eosinophil numbers within groups than seen with the other types of leukocytes studied, such that no significant differences in the mean numbers of alveolar eosinophils was detected between any of the four treatment groups (Fig. 3C).

Leukocyte recruitment markers: ICAM and MCP-1. ICAM-1 was readily detectable in the lung sections (Fig. 4A). In the lungs from animals exposed to $>95 \% \mathrm{O}_{2}$, there was an approximate doubling of ICAM-1 staining in the vessels compared with lung sections from animals exposed to RA (Fig. $4 B$ ). The addition of iNO to the $>95 \% \mathrm{O}_{2}$ exposure prevented the hyperoxia-induced increase in ICAM-1 staining, such that the ICAM-1 staining in the vessels from animals
A

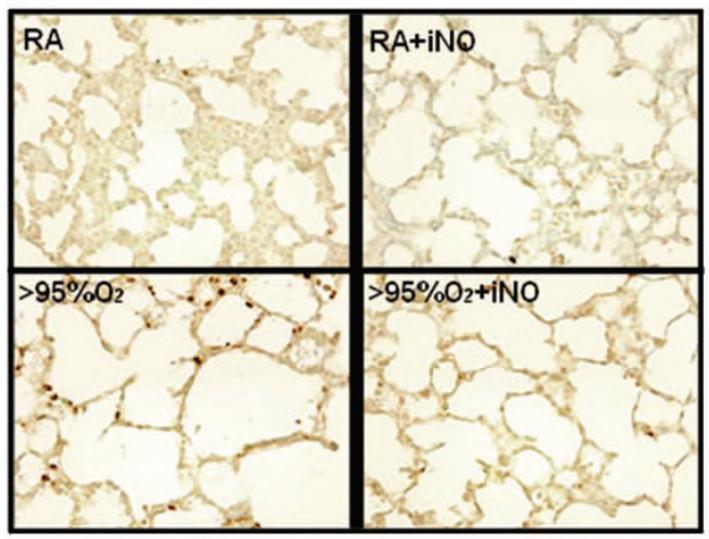

B

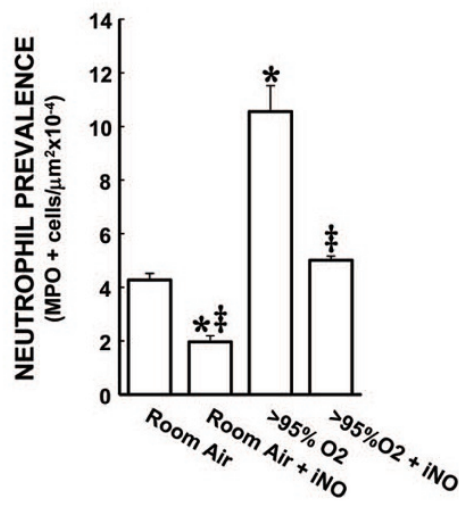

Figure 2. Hyperoxia increased the numbers of neutrophils in neonatal mouse lungs, and the addition of iNO attenuated the hyperoxia-induced increase in neutrophil numbers. A, Representative MPOstained images $(800 \times$ magnification) of alveolar regions from lungs from mice from each of the four treatment groups. $B$, The mean \pm SEM of the neutrophil numbers from all of the lung sections examined. *Different from RA, $p<0.05$. $\neq$ Different from $>95 \% \mathrm{O}_{2}, p<0.05$.
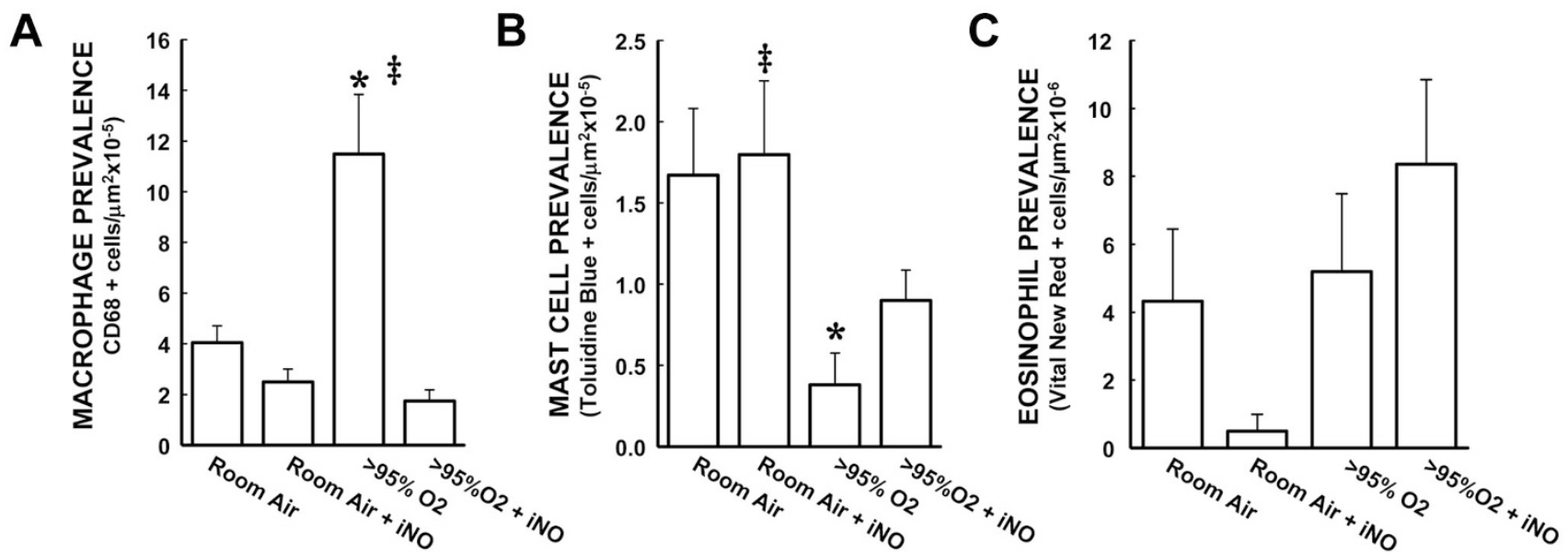

Figure 3. The effect of hyperoxia and iNO on numbers of macrophages, mast cells, and eosinophils in neonatal mouse lungs. A, The mean \pm SEM of the macrophage numbers from all of the sections stained for CD68. There were substantially greater macrophage numbers in the lung sections from $>95 \%$ $\mathrm{O}_{2}$-exposed mice than in those from RA controls, and the addition of iNO to the $>95 \% \mathrm{O}_{2}$ exposure prevented the hyperoxia-induced increase in macrophage numbers. *Different from RA, $p<0.05$. $\ddagger$ Different from $>95 \% \mathrm{O}_{2}, p<0.05 . B$, The mean \pm SEM of the mast cell numbers from all of the lung sections stained with toluidine blue. Hyperoxic exposure significantly decreased mast cell numbers in the lung compared with RA controls, and the addition of iNO to the $>95 \%$ $\mathrm{O}_{2}$ exposure attenuated the hyperoxia-induced decrease in mast cell numbers in the lung. *Different from RA, $p<0.05$. $\ddagger$ Different from $>95 \% \mathrm{O}_{2}, p<0.05$. $C$, The mean \pm SEM of the eosinophil numbers from all of the sections stained with vital new red. There were no significant differences between the four groups in eosinophil numbers in the lung. 
A

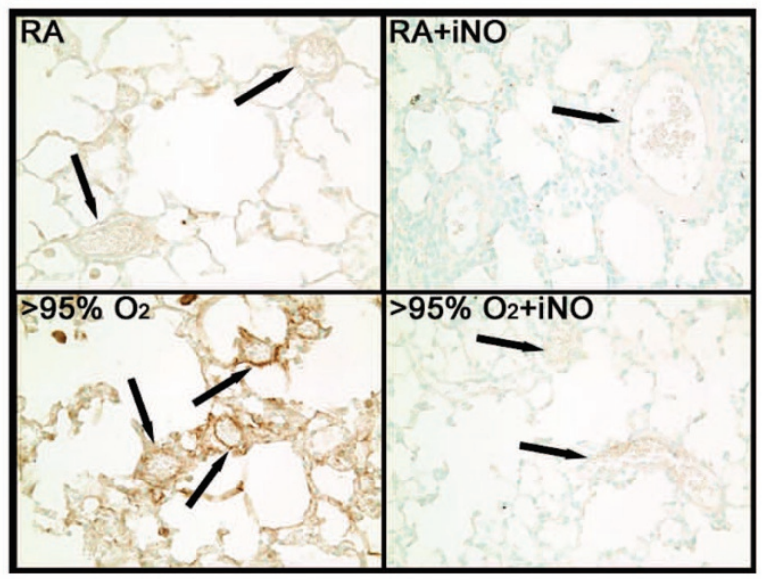

B

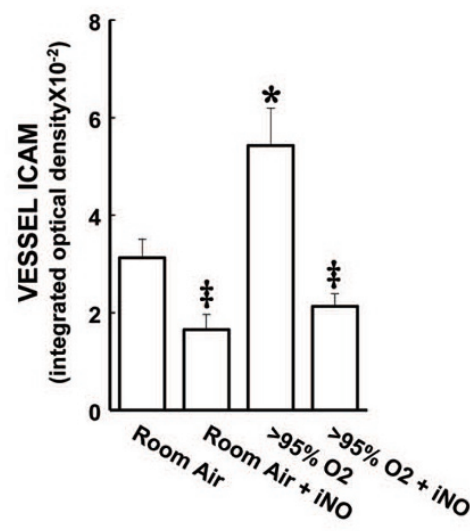

Figure 4. Intracellular adhesion molecule (ICAM)-1 protein levels in the neonatal lung are increased by exposure to hyperoxia, and adding iNO prevents the hyperoxia-induced up-regulation of ICAM protein levels. A, Representative ICAM-1stained images $(800 \times$ magnification) from lungs of mice in each of the four exposure groups. The arrows identify pulmonary vessels. $B$, The mean \pm SEM of the ICAM-1 staining from the vessels from all of the lung sections examined. *Different from RA, $p<0.05$. $\ddagger$ Different from $>95 \% \mathrm{O}_{2}, p<0.05$.

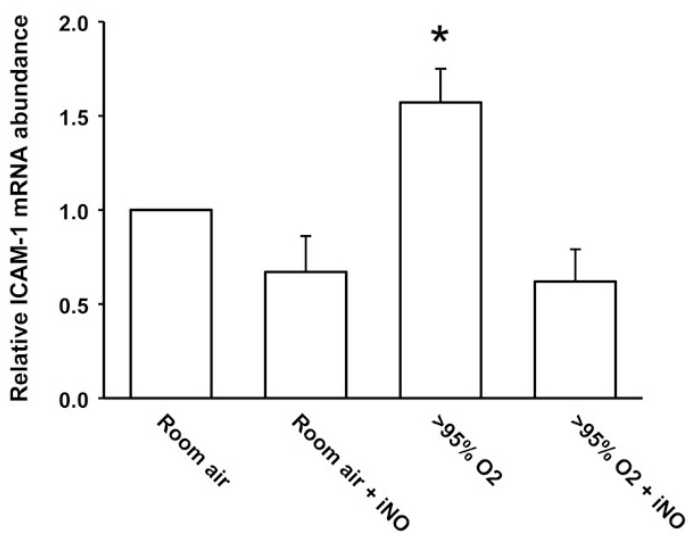

Figure 5. ICAM-1 mRNA levels are increased in the neonatal lung by exposure to hyperoxia and addition of iNO to the hyperoxic exposure prevents the hyperoxia-induced up-regulation of ICAM-1 mRNA levels. Lungs were harvested after $24 \mathrm{~h}$ from animals ( $n=3$ in each group) from each of the four exposure groups. Real-time PCR was done with primers specific for ICAM-1 and normalized to $18 \mathrm{~S}$ rRNA using the $\Delta \Delta \mathrm{Ct}$ method and expressed as fold change from $21 \% \mathrm{O}_{2}$. *Different from RA, $p<0.05$. $\neq$ Different from $>95 \%$ $\mathrm{O}_{2}$ alone, $p<0.05$.

exposed to iNO $+>95 \% \mathrm{O}_{2}$ was not different from ICAM-1 staining in the vessels from animals exposed to RA (Fig. 4B). The addition of iNO to the RA exposure had no significant effect on ICAM-1 staining in the lung vessels (Fig. $4 B$ ). We found that ICAM-1 mRNA was increased by exposure to $>95 \% \mathrm{O}_{2}$ and that this hyperoxia-induced increase was prevented by addition of $\mathrm{NO}$ to the $>95 \% \mathrm{O}_{2}$ exposure (Fig. 5).

A similar pattern of response was observed for MCP-1 staining (Fig. 6). Exposure to hyperoxia resulted in levels of MCP-1 staining that were $\sim 6$-fold greater than that seen in the lungs from animals exposed to RA alone (Fig. 6A). The addition of $10 \mathrm{ppm}$ iNO completely prevented the hyperoxiainduced increase in MCP-1 staining in the lung sections (Fig. $6 A$ ). The addition of iNO to the RA exposure had no significant effect on MCP-1 staining in the lung (Fig. 6A). The expression of MCP-1 mRNA in the whole lung at $24 \mathrm{~h}$ was significantly increased by exposure to $>95 \% \mathrm{O}_{2}$ and the hyperoxia-induced increase in MCP-1 mRNA was prevented by addition of iNO to the $>95 \% \mathrm{O}_{2}$ exposure (Fig. $6 B$ ).
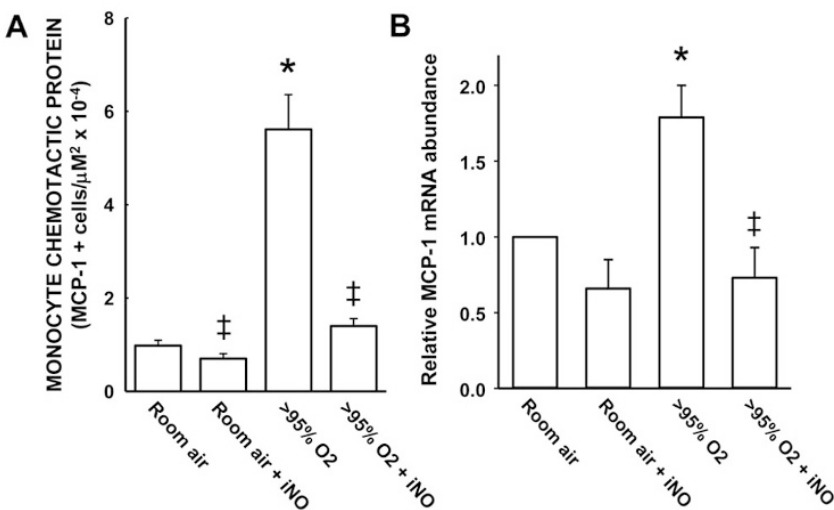

Figure 6. Hyperoxia up-regulates MCP-1 in the lung, whereas the addition of iNO to hyperoxia prevents this response. $(A)$ The mean \pm SEM of the MCP-1 staining from all of the lung sections examined. $(B)$ The mean \pm SEM of the MCP-1 mRNA abundance from real time PCR. *Different from RA, $p<0.05$. Different from $>95 \% \mathrm{O}_{2}, p<0.05$.

Correlation analysis. Univariate comparisons of alveolar number with the other measured variables showed that exposure to $>95 \% \mathrm{O}_{2}$ and neutrophil prevalence were both significantly negatively correlated with alveolar number $(p<0.05$, $\mathrm{r}=-0.69$ and -0.75 , respectively) and significantly correlated with each other $(p<0.05, \mathrm{r}=0.77)$. This indicates that under hyperoxic conditions, more neutrophils were present and both $>95 \% \mathrm{O}_{2}$ and neutrophils resulted in fewer alveoli. A correlation between neutrophil numbers and alveoli was not significant when using the iNO $+>95 \% \mathrm{O}_{2}$ exposure.

\section{DISCUSSION}

The main findings of this study were that: 1) exposure to $>95 \% \mathrm{O}_{2}$ resulted in fewer alveoli, 2) the addition of iNO to the $>95 \% \mathrm{O}_{2}$ exposure prevented the hyperoxia-induced decrease in alveolar number, 3) hyperoxia resulted in the influx of neutrophils and macrophages into the lung, 4) iNO prevented the hyperoxia-induced influx of neutrophils and macrophages into the lung, 5) hyperoxia resulted in increased ICAM and MCP-1 expression in the lungs, and 6) iNO prevented the hyperoxia-induced increase in ICAM and MCP-1 expression. Taken together, these results demonstrate 
that exposure to $>95 \% \quad \mathrm{O}_{2}$ results in decreased alveolar number, increased neutrophil and macrophage influx, and increased lung adhesion molecule protein expression. These results are consistent with our hypothesis and demonstrate that iNO prevents lung adhesion molecule expression and thereby attenuates neutrophil and macrophage influx into hyperoxiaexposed lung tissue.

There are various proposed mechanisms of hyperoxiainduced lung injury, including direct oxidative damage via the generation of free radicals, alterations in vascular endothelial growth factor expression, regulation of chemotactic cytokines, and recruitment of leukocytes (1-4). We found that leukocytes were more prevalent in alveoli under hyperoxic conditions and that the presence of leukocytes in the lung tissue was inversely correlated with our measure of alveolar number. The presence of neutrophils within tracheal effluent or bronchoalveolar lavage fluid has been shown in patients with respiratory distress syndrome who go on to develop BPD (10-12). Interestingly, depleting mice of neutrophils has little effect on hyperoxia-induced lung injury as measured by bronchoalveolar lavage fluid protein concentration (13). On the other hand, blocking neutrophil influx into the lung prevented hyperoxiainduced DNA damage (14) and preserved alveolar development (15) in the lungs of hyperoxia-exposed neonatal rats. We showed that iNO decreased the presence of neutrophils and macrophages within the alveoli and preserved alveolarization in hyperoxia-exposed neonatal mice.

In our study, ICAM expression was up-regulated within the lungs of the hyperoxia group. An increase in ICAM protein levels after hyperoxic exposure has been found in adult mice exposed to hyperoxia and correlated with an increase in MPO staining $(13,16)$. Neonates $<29$-wk gestation who developed BPD were shown in one study to have higher levels of soluble ICAM-1 in the first week of life than those who did not develop BPD (17). MCP-1 protein expression was also increased in our model. In neonatal rat lungs exposed to $>95 \%$ $\mathrm{O}_{2}$, increased influx of macrophages has been found and this was associated with an increase in MCP-1 mRNA expression in the lung (18). Similarly, MCP-1 was increased in neonatal rats exposed to $>95 \% \mathrm{O}_{2}$ and inhibiting MCP-1 using a blocking antibody decreased bronchoalveolar lavage fluid neutrophils and macrophages (19). Therapeutic interventions to reduce inflammatory cell presence and their cytotoxic effects within the lungs, without compromising global neutrophil function are currently being sought, and ICAM and/or MCP-1 may represent targets for this kind of therapeutic intervention.

When iNO was added to the RA exposure, there was greater alveolar number and fewer neutrophils in the lungs then in the lungs of animals exposed to RA alone. There was a tendency for ICAM-1 and MCP-1 expression to decrease when iNO was added to the RA exposure, although these changes did not reach statistically significant. It is possible that iNO has direct effects on alveolarization in the lungs of neonatal mice that does not involve alterations in inflammation or anti-oxidant properties. In adult rabbit lungs, a 6-h exposure to iNO after saline treatment had no effect on lung neutrophil numbers or IL-1 $\beta$ expression (20), suggesting that in RA iNO had little effect on inflammatory markers. There is a paucity of studies examining iNO and alveolarization in neonatal animals in RA; however, in a study on neonatal rats there was no effect of iNO on alveolar number in RA (21). Thus, it remains unclear why in our studies adding iNO to the RA exposure increased alveolar number and decreased neutrophil influx in the lung. Furthermore, NO in the presence of hyperoxia can be metabolized to compounds that can exert either prooxidant or antioxidant effects $(22,23)$. It may be that there is an advantage to adding iNO to RA, because we found a positive effect on lung growth and there would be less chance for the production of prooxidant metabolites of NO, although clinical trials are necessary.

It is important to note that there are limitations in the study design. We studied a relatively small number of genetically inbred animals. Therefore, it is difficult to translate our findings directly to the clinical arena, since patients are genetically diverse and have a wide variety of diseases underlying their need for iNO therapy. We also used a model of hyperoxia in these studies, whereas patients with BPD are usually intubated and receive intermittent positive pressure ventilation for some period of time. These patients are also treated with variable concentrations of supplemental oxygen and, in this study, we used a fixed concentration. Thus, caution must be taken when trying to translate our study to human disease. However, our findings do support the continued development of clinical trials examining iNO and adhesion molecules as therapeutic targets in the prevention of BPD.

We found improvements in the hyperoxia-induced decreased alveolarization in neonatal mice when iNO was added to the hyperoxic exposure. In human studies to date, iNO has shown less clear-cut benefit in preventing BPD $(22,23)$. It may be that our model of hyperoxia-induced arrested alveolarization does not capture all of the factors that underlie the pathogenesis of BPD, such as preterm birth, mechanical ventilation, intrauterine exposure to inflammation, poor nutrition, etc (24). In the clinical setting, BPD may require a more complex interaction of these various factors than exposure to supplemental $\mathrm{O}_{2}$ alone. Alternatively, it may be that the studies to date have failed to show consistent reductions in BPD incidence due to timing of the initiation of iNO, the dose of iNO, and/or the duration of iNO $(22,23)$. Finally, it is important to note that BPD is defined by a requirement for supplemental $\mathrm{O}_{2}$ therapy after preterm birth (25). Thus, it may be that BPD is actually several different lung phenotypes, including but not limited to decreased alveolarization.

In summary, exposure to hyperoxia resulted in a decrease in alveolar number in the neonatal mouse lung, and exposure to hyperoxia and iNO prevented the hyperoxia-induced decrease in alveolar number. We found that hyperoxia resulted in up-regulation of ICAM-1 and MCP-1 in the neonatal lungs, which resulted in the recruitment of neutrophils and macrophages to the lungs. This pattern of hyperoxia-induced adhesion molecule expression and neutrophil and macrophage recruitment was prevented by adding iNO to the hyperoxic exposure. Up-regulation of adhesion molecule expression in the lung by hyperoxia may represent a novel site of action of 
iNO in vivo that could direct further clinical therapeutics to reduce the incidence of chronic lung disease in neonates.

Acknowledgments. We thank Dionna Hatch and Yi Jin for excellent technical assistance.

\section{REFERENCES}

1. Deng H, Mason SN, Auten RL Lung inflammation in hyperoxia can be prevented by antichemokine treatment in newborn rats. Am J Respir Crit Care Med 162:23162323

2. Issa A, Lappalainen U, Kleinman M, Bry K, Hallman M 1999 Inhaled nitric oxide decreases hyperoxia-induced surfactant abnormality in preterm rabbits. Pediatr Res 45:247-254

3. Lin HC, Wang CH, Yu CT, Huang KS, Liu CY, Yang CW, Kuo HP 2003 Effect of endogenous nitric oxide on hyperoxia and tumor necrosis factor-alpha-induced leukosequestration and proinflammatory cytokine release in rat airways. Crit Care Med 31:508-516

4. Lin YJ, Markham NE, Balasubramaniam V, Tang JR, Maxey A, Kinsella JP, Abman SH 2005 Inhaled nitric oxide enhances distal lung growth after exposure to hyperoxia in neonatal rats. Pediatr Res 58:22-29

5. Park MS, Rieger-Fackeldey E, Schanbacher BL, Cook AC, Bauer JA, Rogers LK, Hansen TN, Welty SE, Smith CV 2007 Altered expressions of fibroblast growth factor receptors and alveolarization in neonatal mice exposed to $85 \%$ oxygen. Pediatr Res 62:652-657

6. Weinberger B, Heck DE, Laskin DL, Laskin JD 1999 Nitric oxide in the lung: therapeutic and cellular mechanisms of action. Pharmacol Ther 84:401-411

7. 1997 Inhaled nitric oxide in full-term and nearly full-term infants with hypoxic respiratory failure. The Neonatal Inhaled Nitric Oxide Study Group. N Engl J Med 336: 597-604

8. Clark RH, Kueser TJ, Walker MW, Southgate WM, Huckaby JL, Perez JA, Roy BJ, Keszler M, Kinsella JP 2000 Low-dose nitric oxide therapy for persistent pulmonary hypertension of the newborn. N Engl J Med 342:469-474

9. Kinsella JP, Abman SH 2007 Inhaled nitric oxide in the premature newborn. J Pediatr 151:10-15

10. Merritt TA, Cochrane CG, Holcomb K, Bohl B, Hallman M, Strayer D, Edwards DK, Gluck L 1983 Elastase and alpha 1-proteinase inhibitor activity in tracheal aspirates during respiratory distress syndrome. Role of inflammation in the pathogenesis of bronchopulmonary dysplasia. J Clin Invest 72:656-666
11. Ogden BE, Murphy S, Saunders GC, Johnson JD 1983 Lung lavage of newborns with respiratory distress syndrome. Prolonged neutrophil influx is associated with bronchopulmonary dysplasia. Chest 83:31S-33S

12. Speer CP, Ruess D, Harms K, Herting E, Gefeller O 1993 Neutrophil elastase and acute pulmonary damage in neonates with severe respiratory distress syndrome. Pediatrics 91:794-799

13. Perkowski S, Scherpereel A, Murciano JC, Arguiri E, Solomides CC, Albelda SM, Muzykantov V, Christofidou-Solomidou M 2006 Dissociation between alveolar transmigration of neutrophils and lung injury in hyperoxia. Am J Physiol Lung Cell Mol Physiol 291:L1050-L1058

14. Auten RL, Whorton MH, Mason SN 2002 Blocking neutrophil influx reduces DNA damage in hyperoxia-exposed newborn rat lung. Am J Respir Cell Mol Biol 26:391-397

15. Auten RL, Mason SN, Tanaka DT, Welty-Wolf K, Whorton MH 2001 Antineutrophil chemokine preserves alveolar development in hyperoxia-exposed newborn rats. Am J Physiol Lung Cell Mol Physiol 281:L336-L344

16. Welty SE, Rivera JL, Wu B 1997 Hyperoxic increases in lung ICAM-1 mRNA are independent of TNF-alpha and IL-1 beta mRNA. Free Radic Biol Med 23:898-908

17. Ramsay PL, O’Brian Smith E, Hegemier S, Welty SE 1998 Early clinical markers for the development of bronchopulmonary dysplasia: soluble E-Selectin and ICAM-1. Pediatrics 102:927-932

18. ter Horst SA, Walther FJ, Poorthuis BJ, Hiemstra PS, Wagenaar GT 2007 Inhaled nitric oxide attenuates pulmonary inflammation and fibrin deposition and prolongs survival in neonatal hyperoxic lung injury. Am J Physiol Lung Cell Mol Physiol 293:L35-L44

19. Vozzelli MA, Mason SN, Whorton MH, Auten RL 2004 Antimacrophage chemokine treatment prevents neutrophil and macrophage influx in hyperoxia-exposed newborn rat lung. Am J Physiol Lung Cell Mol Physiol 286:L488-L493

20. Kang JL, Park W, Pack IS, Lee HS, Kim MJ, Lim CM, Koh Y 2002 Inhaled nitric oxide attenuates acute lung injury via inhibition of nuclear factor-kappa B and inflammation. J Appl Physiol 92:795-801

21. Tang JR, Markham NE, Lin YJ, McMurtry IF, Maxey A, Kinsella JP, Abman SH 2004 Inhaled nitric oxide attenuates pulmonary hypertension and improves lung growth in infant rats after neonatal treatment with a VEGF receptor inhibitor. Am J Physiol Lung Cell Mol Physiol 287:L344-L351

22. Arul N, Konduri GG 2009 Inhaled nitric oxide for preterm neonates. Clin Perinatol $36: 43-61$

23. Barrington KJ, Finer NN 2007 Inhaled nitric oxide for preterm infants: a systematic review. Pediatrics 120:1088-1099

24. Donn SM, Sinha SK 2006 Minimising ventilator induced lung injury in preterm infants. Arch Dis Child Fetal Neonatal Ed 91:F226-F230

25. Ehrenkranz RA, Walsh MC, Vohr BR, Jobe AH, Wright LL, Fanaroff AA, Wrage LA, Poole K; National Institutes of Child Health and Human Development Neonatal Research Network 2005 Validation of the National Institutes of Health consensus definition of bronchopulmonary dysplasia. Pediatrics 116:1353-1360 